Research article

\title{
Phi class glutathione transferases as molecular targets towards multiple-herbicide resistance: Inhibition analysis and pharmacophore design
}

\author{
Nikolaos Georgakis $^{\mathrm{a}}$, Nirmal Poudel ${ }^{\mathrm{b}}$, Dimitrios Vlachakis ${ }^{\mathrm{c}}$, Anastassios C. Papageorgiou ${ }^{\mathrm{b}}$, \\ Nikolaos E. Labrou ${ }^{\text {a,* }}$ \\ ${ }^{\text {a }}$ Laboratory of Enzyme Technology, Department of Biotechnology, School of Applied Biology and Biotechnology, Agricultural University of Athens, 75 Iera Odos Street, \\ GR, 11855, Athens, Greece \\ b Turku Bioscience Centre, University of Turku and Åbo Akademi University, Turku, 20521, Finland \\ ${ }^{\mathrm{c}}$ Laboratory of Genetics, Department of Biotechnology, School of Applied Biology and Biotechnology, Agricultural University of Athens, 75 Iera Odos Street, GR, 11855 , \\ Athens, Greece
}

\section{A R T I C L E I N F O}

\section{Keywords:}

Abiotic stress

Biotic stress

Glutathione transferase

Xenobiotic metabolism

\begin{abstract}
A B S T R A C T
Multiple-herbicide resistance (MHR) is a global threat to weed control in cereal crops. MHR weeds express a specific phi class glutathione transferase (MHR-GSTF) that confers resistance against multiple herbicides and therefore represents a promising target against MHR weeds. Kinetics inhibition analysis of MHR-GSTFs from grass weeds Lolium rigidum (LrGSTF) Alopecurus myosuroides (AmGSTF) and crops Hordeum vulgare (HvGSTF) and Triticum aestivum (TaGSTF) allowed the identification of the acetanilide herbicide butachlor as a potent and selective inhibitor towards MHR-GSTFs. Also, butachlor is a stronger inhibitor for LrGSTF and AmGSTF compared to $H \nu$ GSTF and TaGSTF from crops. The crystal structure of LrGSTF was determined at $1.90 \AA$ resolution in complex with the inhibitor S-(4-nitrobenzyl)glutathione. A specific 3D pharmacophore targeting the MHR-GSTFs was designed and used to identify structural elements important for potent and selective inhibition. Structural analysis of GSTFs revealed a decisive role of conserved Tyr118 in ligand binding and pharmacophore design. Its positioning is dependent on an outer patch of adjacent residues that span from position 132 to 134 which are similar for both $L r G S T F$ and AmGSTF but different in HvGSTF and TaGSTF. The results presented here provide new knowledge that may be adopted to cope with MHR weeds.
\end{abstract}

\section{Introduction}

As more knowledge on structure, phylogeny, gene expression patterns and both catalytic and non-catalytic functions of GSTs are being elucidated, their biological roles are extended beyond xenobiotic detoxification (Axarli et al., 2009a, 2009b; Benekos et al., 2010; Dixon et al., 2010; Dixon and Edwards, 2009; Zhao, 2015). The biological roles of GSTs have been connected with tolerance to numerous abiotic and biotic stress factors as well as cell growth, regulation of cellular redox state (Axarli et al., 2009a, 2009b; Benekos et al., 2010; Dixon et al., 2010; Dixon and Edwards, 2009; Zhao, 2015), transport of anthocyanins, flavonoids, oxylipins, phenolics, haemins (Sylvestre-Gonon et al., 2020), and hormones such as auxin and cytokinin, suggesting a possible role in cell signaling (Dixon et al., 2011).
Plant GSTs have been identified as major mediators of metabolic resistance to herbicides through their catalytic activity to conjugate the tripeptide glutathione (GSH) to a wide range of herbicides (Busi et al., 2018; Gaines et al., 2020; Gao et al., 2019; Nakka et al., 2017). A specific phi class glutathione transferase (MHR-GSTF) that also exhibits high glutathione peroxidase activity has been identified as a major factor (Cummins et al., 1999, 2013; Georgakis et al., 2020; Pégeot et al., 2017) that confers resistance against multiple herbicides through multiple mechanisms (Tétard-Jones et al., 2018). Because of the rapid spread of MHR in weeds and the restricted development of new herbicides, the identification of synergistic compounds which when combined with the existing herbicides can inhibit MHR-GSTF and therefore restore weed control in MHR weeds would certainly be an important development. Such inhibiting compounds, can offer a viable alternative strategy in counteracting resistance in the field. Similar approaches have already

\footnotetext{
* Corresponding author.

E-mail address: lambrou@aua.gr (N.E. Labrou).
} 


\author{
Abbreviations \\ AmGSTF GSTF from Alopecurus myosuroides \\ Butachlor [N-(butoxymethyl)-2-chloro-N-2,6-dimethyl \\ acetanilide] \\ GSH glutathione \\ GST glutathione transferase \\ GS8 S-hydroxy-GSH \\ G-site GSH binding site \\ HvGSTF GSTF from Hordeum vulgare \\ LrGSTF GSTF from Lolium rigidum \\ MHR multiple-herbicide resistance \\ Nb-GSH S-(4-nitrobenzyl)glutathione \\ H-site hydrophobic binding site \\ SIN Succinic acid \\ TaGSTF GSTF from Triticum aestivum \\ WTS wild-type sensitive
}

been developed. For example, piperonyl butoxide that inhibits the detoxification enzymes cytochrome $\mathrm{P} 450$ and esterases has been used to counteract metabolic-based insecticide resistance (Karatolos et al., 2012). Similarly, 4-chloro-7-nitro-benzoxadiazole inhibits MHR-GSTF activity and helps restore herbicide control in MHR black-grass (Cummins et al., 2013).

The phi class GSTs are biologically active as dimers (Ahmad et al., 2017; Dobritzsch et al., 2020; Pégeot et al., 2014). Each subunit has an independent active site that is formed by two distinct sub-sites: the GSH-binding site (G-site) in the N-terminal domain and an electrophile substrate-binding site (H-site) in the C-terminal domain as in other GST classes (Axarli et al., 2009a, 2009b; Neuefeind et al., 1997). The $\mathrm{N}$-terminal domain is conserved among different isoenzymes, whereas the $\mathrm{C}$-terminal domain is quite variable, thus leading to distinct specificities toward the xenobiotic substrate (Axarli et al., 2009a, 2009b; Dixon et al., 2010; Dixon and Edwards, 2009; Georgakis et al., 2020).

In the present work, tested pesticides are listed in EU - Pesticides database (e.g. EU - https://ec.europa.eu/food/plant/pesticides/eu-pest icides-database). All listed pesticides have known and wellcharacterized toxicity, bioactivity, safety and bioavailability - properties which could dramatically accelerate inhibitor development and optimization. The evaluation of such pesticides for this purpose may yield new activities unrelated to their intended use and may hasten the discovery of new GST-specific inhibitors.

\section{Materials and methods}

\subsection{Materials}

Reduced GSH, 1-chloro-2,4-dinitrobenzene (CDNB), S-(p-nitrobenzyl)-glutathione, analytical grade pesticides and natural products used for inhibition studies were obtained from Sigma-Aldrich, (USA).

\subsection{Methods}

\subsubsection{Enzyme expression and purification}

Enzymes (LrGSTF, NCBI accession number: CCO25537.1; AmGSTF, NCBI accession number: CAA09192.1; HvGSTF, NCBI accession number: AAL73394.1; TaGSTF, NCBI accession number: AAL47688.1) were cloned, expressed in $E$. coli and purified by affinity chromatography as described by Georgakis et al. (2020).

\subsubsection{Screening of pesticide, natural product and S-alkyl-GSH-inhibitor} libraries as possible inhibitors against GSTFs

Enzyme assays were carried out according to Georgakis et al. (2020).
The inhibition potency of different pesticides was assayed at $25 \mu \mathrm{M}$ concentration, using the CDNB-GSH assay system $\left(25^{\circ} \mathrm{C}\right)$. The enzyme inhibition assays contained, in a total volume of $1 \mathrm{~mL}$ : potassium phosphate buffer ( $100 \mathrm{mM}, \mathrm{pH}$ 6.5), an appropriate concentration of GSH (2.50 mM for AmGSTF, $1.83 \mathrm{mM}$ for LrGSTF, $2.50 \mathrm{mM}$ for $H \nu \mathrm{GSTF}$ and $1.95 \mathrm{mM}$ for TaGSTF, prepared in water), $25 \mu \mathrm{M}$ inhibitor and enzyme (5-50 $\mu \mathrm{L}$ of purified GST, typically producing $0.11 \Delta \mathrm{A}_{340}$ per min). The mixture was incubated at $25{ }^{\circ} \mathrm{C}$ for $1 \mathrm{~min}$, before adding CDNB. The concentration of CDNB was adjusted to equal the $\mathrm{K}_{\mathrm{m}(\mathrm{CDNB})}$ value (Georgakis et al., 2020) of the examined enzyme (1.0 mM for AmGSTF, $0.73 \mathrm{mM}$ for $L r G S T F, 1.0 \mathrm{mM}$ for $H \nu \mathrm{GSTF}$ and $0.78 \mathrm{mM}$ for TaGSTF). The observed reaction rates were used to calculate the remaining activity (\%) taking as $100 \%$ the initial enzyme activity determined in the absence of inhibitor. All pesticides were dissolved in acetone; natural products were dissolved in ethanol, DMSO or diluted $\mathrm{NaOH}$, and S-alkyl-GSH-Inhibitors were dissolved in water, $\mathrm{KH}_{2} \mathrm{PO}_{4}$, DMSO, ethanol or acetonitrile. $\mathrm{IC}_{50}$ values were determined using the GraphPad Prism version 7. The Bradford assay (Bradford, 1976) was used for measuring protein concentration.

\subsubsection{Crystallization and structure determination}

LrGSTF was concentrated to $\sim 15 \mathrm{mg} / \mathrm{ml}$ in $10 \mathrm{mM}$ HEPES buffer, $100 \mathrm{mM} \mathrm{NaCl}, \mathrm{pH}$ 7.0. Crystallizations were initially setup with five commercial crystallization screens in 96-well plates at $20^{\circ} \mathrm{C}$. Crystals in the presence of S-(4-nitrobenzyl)glutathione $(10 \mathrm{mM})$ were found in condition 21 (20\% w/v Sokalan PA 25CL, 0.1 M HEPES buffer pH 7.0, 0.1 M Sodium tartrate) of the MIDASplus screen (Molecular Dimensions). Data were collected to $1.90 \AA$ resolution with a total exposure time of $28 \mathrm{~s}$ at $0.9762 \AA$ wavelength and an exposure time per frame of $0.021 \mathrm{~s}$ (total number of frames 1333) using an EIGER 16M detector at the P14 beamline (PETRA III, DESY, Hamburg, Germany) under cryogenic temperatures with $20 \%$ glycerol as cryoprotectant. The space group was $P 2_{1}$ and the cell parameters $92.6 \AA$, $98.5 \AA$, $95.9 \AA$, $\beta=$ $109.1^{\circ}$, suggesting 6 molecules in the asymmetric unit based on solvent content considerations (Matthews, 1968). Data processing was carried out with XDS (Kabsch, 2010) followed by merging and scaling with AIMLESS (Evans and Murshudov, 2013) through the EDNA pipeline (Incardona et al., 2009). The structure was determined by molecular replacement using PHASER (McCoy et al., 2007) as implemented in PHENIX v. 1.17.1-3660 (Adams et al., 2010). A search model of a Zea mays GST (PDB id 1bye, $62.4 \%$ seq. identity) was constructed with SCULPTOR (Bunkoczi and Read, 2011). Six molecules of LrGSTF were located and the resulting solution was further explored for rebuilding and refinement. The structure was refined with PHENIX to final $R_{\text {work }} / R_{\text {free }}$ of $0.165 / 0.203$. A molecule of [S-(p-nitrobenzyl)-glutathione] (Nb-GSH) was added in five $L r$ GSTF subunits based on the $F_{\mathrm{o}}-F_{\mathrm{c}}$ electron density map. A molecule of GSH was added in subunit D. Of all LrGSTF molecules, C displays electron density for all residues, including the $\mathrm{C}$-terminal helix and the six His residues of the purification tag. Data collection and refinement statistics are given in Table 2.

\subsubsection{Biocomputing analysis}

Homology modelling of HvGSTF and TaGSTF. The LrGSTF structure was used as a template for $\mathrm{H} \nu \mathrm{GSTF}$ and TaGSTF. The sequence identity between the two GSTs to be modeled and the template was higher than 80 percent, which allowed for conventional homology modelling techniques to be considered. The reliability and quality of the models and the model complexes to docked Nb-GSH, was initially evaluated within the

Table 1

Determination of inhibition potency, $\left(\mathrm{IC}_{50}\right)$, of butachlor towards purified $H \nu$ GSTF, TaGSTF, LrGSTF and AmGSTF.

\begin{tabular}{lllll}
\hline Inhibitor $(\mu \mathrm{M})$ & $H \nu \mathrm{GSTF}$ & TaGSTF & LrGSTF & AmGSTF \\
\hline Butachlor & $30.5 \pm 0.8$ & $41.8 \pm 1.3$ & $7.2 \pm 0.1$ & $7.7 \pm 0.3$ \\
\hline
\end{tabular}


Table 2

$\mathrm{X}$-ray data collection and refinement statistics (numbers in parentheses refer to outer resolution shell).

\begin{tabular}{|c|c|}
\hline & LrGSTF \\
\hline \multicolumn{2}{|l|}{ Data collection } \\
\hline Wavelength $(\AA)$ & 0.9762 \\
\hline Resolution range ( $(\AA)$ & $66.68-1.90(1.93-1.90)$ \\
\hline Space group & $P 2_{1}$ \\
\hline Unit cell $\left(\AA{ }^{\circ},{ }^{\circ}\right)$ & 92.698 .595 .990109 .190 \\
\hline Total reflections & $486200(18253)$ \\
\hline Unique reflections & $126068(5801)$ \\
\hline Multiplicity & $3.9(3.1)$ \\
\hline Completeness (\%) & $98.6(91.5)$ \\
\hline Mean I/sigma(I) & $7.4(1.4)$ \\
\hline Wilson B-factor $\left(\AA^{2}\right)$ & 21.15 \\
\hline$R_{\text {meas }}$ & $0.113(0.862)$ \\
\hline$R_{\text {pim }}$ & $0.076(0.583)$ \\
\hline $\mathrm{CC} 1 / 2$ & $0.997(0.843)$ \\
\hline $\mathrm{CC}^{*}$ & $1(0.963)$ \\
\hline \multicolumn{2}{|l|}{ Refinement } \\
\hline Reflections used in refinement & 125811 (6089) \\
\hline Reflections used for R-free & $1829(134)$ \\
\hline$R_{\text {work }} / R_{\text {free }}$ & $0.165(0.281) / 0.203(0.315)$ \\
\hline $\mathrm{CC}_{\text {work }} / \mathrm{CC}_{\text {free }}$ & $0.971(0.893) / 0.940(0.886)$ \\
\hline Number of non-hydrogen atoms & 11820 \\
\hline Macromolecules & 10508 \\
\hline Ligands & 239 \\
\hline Solvent & 1073 \\
\hline Protein residues & 1325 \\
\hline RMSD in bonds ( $\mathrm{A})$ & 0.006 \\
\hline RMSD in angles $\left(^{\circ}\right)$ & 0.81 \\
\hline Ramachandran favored/allowed/outliers (\%) & $96.84 / 2.77 / 0.39$ \\
\hline Rotamer outliers (\%) & 0.00 \\
\hline Clashscore & 3.55 \\
\hline Average B-factor $\left(\AA^{2}\right)$ & 36.28 \\
\hline Macromolecules & 35.64 \\
\hline Ligands & 41.35 \\
\hline Solvent & 41.57 \\
\hline Number of TLS groups & 6 \\
\hline PDB id & 6zb6 \\
\hline
\end{tabular}

Gromacs (Hess et al., 2008) package by a residue packing quality function, which depends on the number of buried non-polar side chain groups and hydrogen bonding. Moreover, the suite PROCHECK (Laskowski et al., 1996) was employed to further evaluate the quality of the two modeled GSTs. Verify3D (Eisenberg et al., 1997) was also used to evaluate whether the models have similar fold to known protein structures. Finally, the Molecular Operating Environment ((MOE), 2020 http://www.chemcomp.com) suite was used to evaluate the 3D geometry of the models in terms of their Ramachandran plots, omega torsion profiles, phi/psi angles, planarity, C-beta torsion angles and rotamer strain energy profiles.

Energy Minimization and Molecular Dynamics Simulations. Energy minimizations were used to remove any residual geometrical strain in each molecular system, using the Charmm27 forcefield as it is implemented into the Gromacs suite, version 4.5.5 (Hess et al., 2008). The molecular systems were then subjected to unrestrained Molecular Dynamics Simulations (MDS) using the Gromacs suite, version 4.5.5 (Hess et al., 2008). MDS took place in a SPC water-solvated, periodic environment. Water molecules were added using the truncated octahedron box extending $7 \AA$ from each atom. The molecular systems were neutralized with counter-ions as required. For this study, all MDSs were performed using the NVT ensemble in a canonical environment, at $300 \mathrm{~K}$ and a step size equal to 2 fs for a total 100 ns simulation time.

Molecular docking. In order to in silico establish the structures of each GST system in complex with the corresponding compounds, the docking suite ZDOCK (version 3.0) was used (Chen et al., 2003). Docking experiments were conducted on the models that had been energetically minimized and conformationally optimized using molecular dynamics simulations. ZDOCK uses a scoring function that returns electrostatic, hydrophobic and desolvation energies as well as performing a fast pairwise shape complementarity evaluation.

Hybrid QSAR Analysis and Pharmacophore Elucidation. The aim of this analysis was initially to interpret the unique characteristics of various compounds in regards to their estimated activities and to provide the means required to establish a 3D-pharmacophore model that would enable us to more accurately screen for GST specific agents. The predicted inhibitory potential of those compounds was associated with their in vitro activities and linked to the docking conformations. This correlation was subsequently submitted to the Pharmacophore Elucidation Query module of Molecular Operating Environment (MOE), 2020 (http ://www.chemcomp.com). The algorithm initially identifies all features common to the highest-ranking compounds, as well as features present in the least ranking compounds. The first ones are retained, whereas the latter are discarded. Finally, a set of regression parameters is used to estimate the activity value of each compound in the training set. The relationship between the geometric fit value and activity value is utilized for this computation.

\section{Results and discussion}

\subsection{Inhibition analysis}

It is well established that GSTs display extensive "druggability" (ligandin function) (Ahmad et al., 2017; Sylvestre-Gonon et al., 2019) i. e. ability to bind with high affinity to small molecules (Neuefeind et al., 1997; Skopelitou et al., 2017). In order to probe the "druggability" of MHR-GSTFs ( $H v$ GSTF, TaGSTF, AmGSTF and LrGSTF), the inhibition potency of a collection of a wide range of pesticides (herbicides, insecticides and fungicides, Fig. 1), natural products (flavonoids, polyphenols and plant hormones, Fig. 2) and a range of different S-alkyl-GSH analogues with diverse structural features (polarity, hydrophobicity) and size, was evaluated (Fig. 3). In general, the binding of non-substrate ligands to GSTs leads to inhibitory effects on the enzyme activity (Chronopoulou et al., 2012).

Generally, GSTFs possess a smaller H-site cleft compared to other plant GSTs (e.g. tau class). Hence, GSTFs are able to bind smaller, although structurally diverse compounds (Axarli et al., 2009a, 2009b; Neuefeind et al., 1997). For instance, the isoenzymes GSTF2 and GSTF12 from Arabidopsis thaliana have been shown to bind heterocyclic ligands (Ahmad et al., 2017; Dixon et al., 2011) and anthocyanins (Kitamura et al., 2010), respectively. As indicated in Fig. 1, the enzymes HvGSTF, TaGSTF, AmGSTF and LrGSTF are sensitive to inhibition (inhibition > $40 \%$ ) by a very narrow range of pesticides. Bulky pesticides with two or three aromatic rings displayed low inhibition potency. On the other hand, smaller pesticides with one aromatic ring (e.g. chloroacetanilides) exhibited higher inhibition potency or moderate in case of triazoles (e.g. prothioconazole). From a total of 60 pesticides tested, the chloroacetanilide herbicide butachlor demonstrated the highest inhibition potency against all GSTFs. The effectiveness of butachlor, in terms of $\mathrm{IC}_{50}$, against all GSTFs was determined (Fig. 4) and the results are listed in Table 1. The analysis showed that AmGSTF and LrGSTF display higher affinity towards butachlor in comparison with TaGSTF and HvGSTF.

The inhibition of GSTFs by a diverse range of natural products (polyphenols, flavonoids, plant hormones) was also assessed (Fig. 2). The results showed that quercetin and ellagic acid display the highest inhibition potency (inhibition $>70 \%$ ), whereas other flavonoids and polyphenols such as epigallocatechin gallate, resveratrol, curcumin and piperlongumine display lower potency (inhibition $>40 \%$ ). Plant hormones (e.g. gibberellic acid, indole-3-acetic acid, indole-3-butyric acid, kinetin) showed superficial inhibition (0-13\%). These results are in agreement with earlier published investigations. For example, quercetin and naringenin display a moderate-weak inhibition potency when tested against recombinant GSTFs from Triticum aestivum (TaGSTFs) (Cummins et al., 2003). On the other hand, p-coumaric acid, quercetin and naringenin proved incapable of inhibiting GSTF1 from Zea mays (ZmGSTF1). Noteworthy, ZmGSTF1 is sensitive to inhibition by the plant 


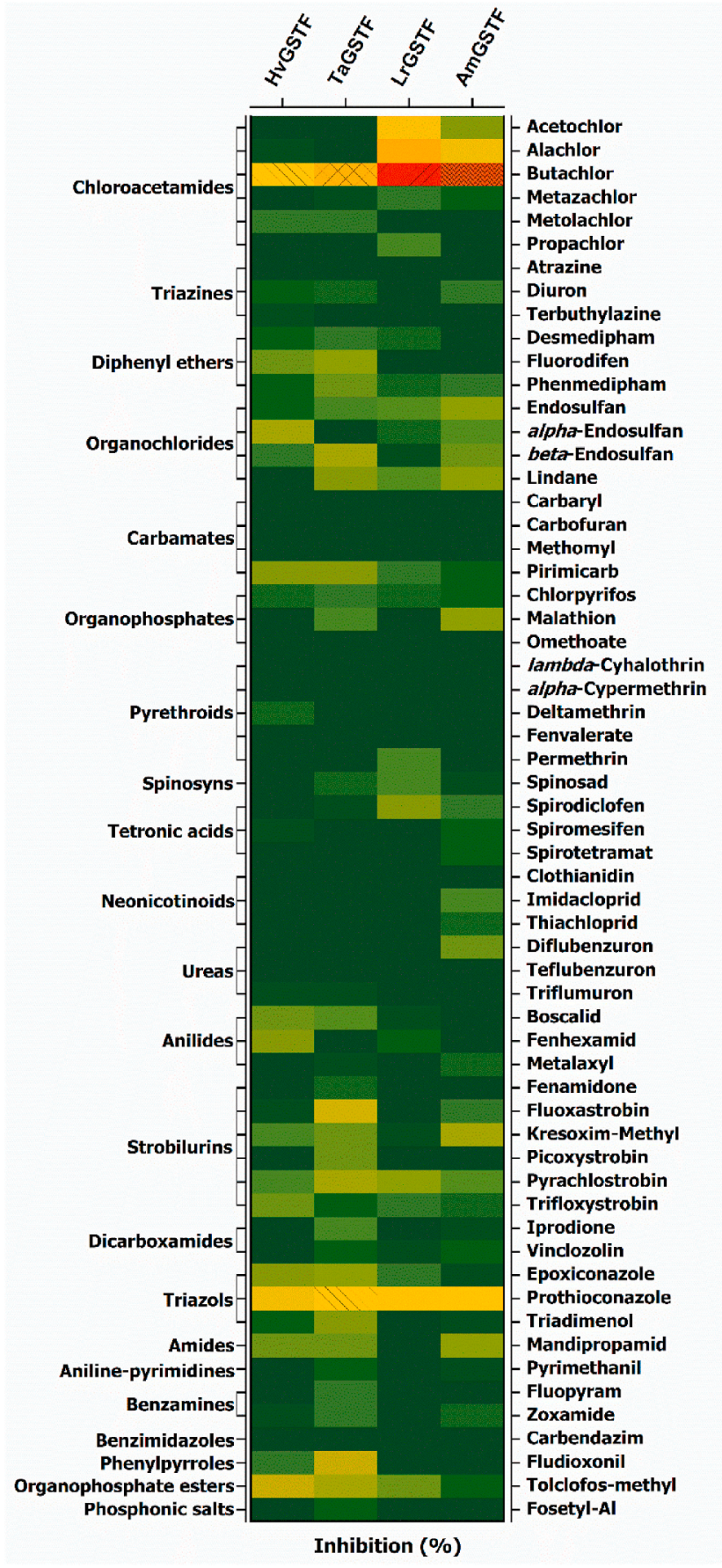

hormones gibberellic acid and 2-nafthoxyacetic acid when assessed by affinity labelling experiments (Axarli et al., 2004). In another example, it has been demonstrated that indole-3-acetic acid is ineffective inhibitor when tested against GSTF5 from Oryza sativa (OsGSTF5) (Cho et al., 2007). More recently, AtGSTF2, expressed in transgenic Nicotiana benthamiana, was found to bind GSH derivatives of fatty acid oxylipin, kaempferol and quercetin at a ratio of a single molecule per AtGSTF dimer, indicating a very specific interaction with flavonoids (Dixon and Edwards, 2018).

\subsection{Description and analysis of LrGSTF crystal structure}

We have recently reported the crystallization and crystal structure analysis of AmGSTF. To gain a deeper knowledge of the structural and
Fig. 1. Inhibition potency of selected pesticides towards $H v G S T F, T a G S T F, L r G S T F$ and AmGSTF. The enzyme assays were implemented in triplicate employing the GSH/CDNB system. The colors represent the mean values of three inhibition assays (\%) for each pesticide against the tested enzymes with variation of less than $5 \%$ in all cases. The motifs (left) allow the discrimination for specific inhibition potency according to the table legend (right). (For interpretation of the references to color in this figure legend, the reader is referred to the Web version of this article.) functional relationship of the phi class GSTs, the crystal structure of LrGSTF was resolved for the first time and characterized (Fig. 5A and B). LrGSTF was crystallized with six molecules in the asymmetric unit. Each of the six LrGSTF molecules (A, B, C, D, E and F) have 222, 215, 227, 215, 215 and 215 amino acid residues built in the structure, respectively, owing to flexibility at the C-terminal and the lack of adequate electron density. Molecule $\mathrm{C}$ exhibits the lowest flexibility, resulting in the location of the entire $\mathrm{C}$-terminal $6 \mathrm{xHis}$-tag. The typical dimers found in GSTs are formed by molecules F and C, A and E (Fig. 5A and B). The LrGSTF monomer comprises an N-terminal thioredoxin-like $\left(\beta_{1} \alpha_{1} \beta_{2} \alpha_{2} \beta_{3} \beta_{4} \alpha_{3}\right)$ domain and an all-helical C-terminal domain $\left(\alpha_{4} \alpha_{5} \alpha_{6} \alpha_{6} \alpha_{7} \alpha_{8}\right)$. A large open active-site cavity is located between the $C$ and N-terminal domains. The N-terminal domain houses the G-site for GSH binding and is highly conserved. Similarly, the C-terminal domain 


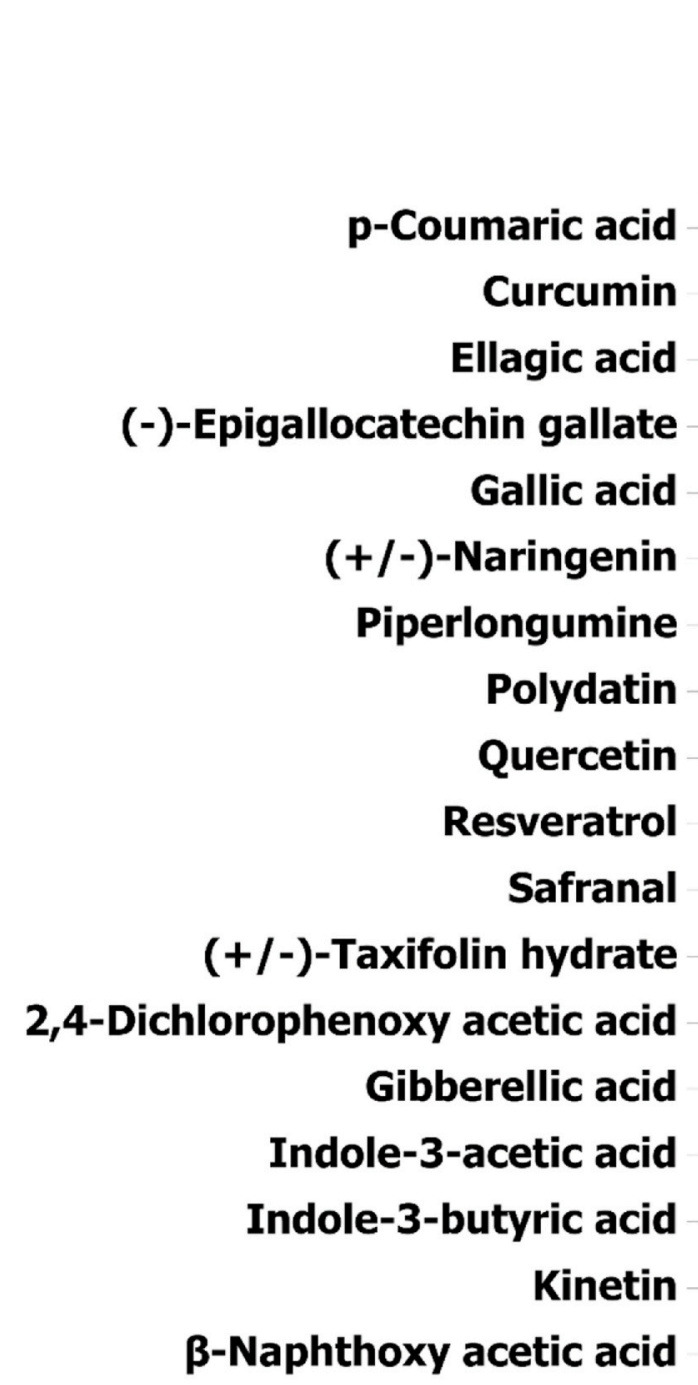

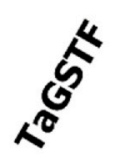
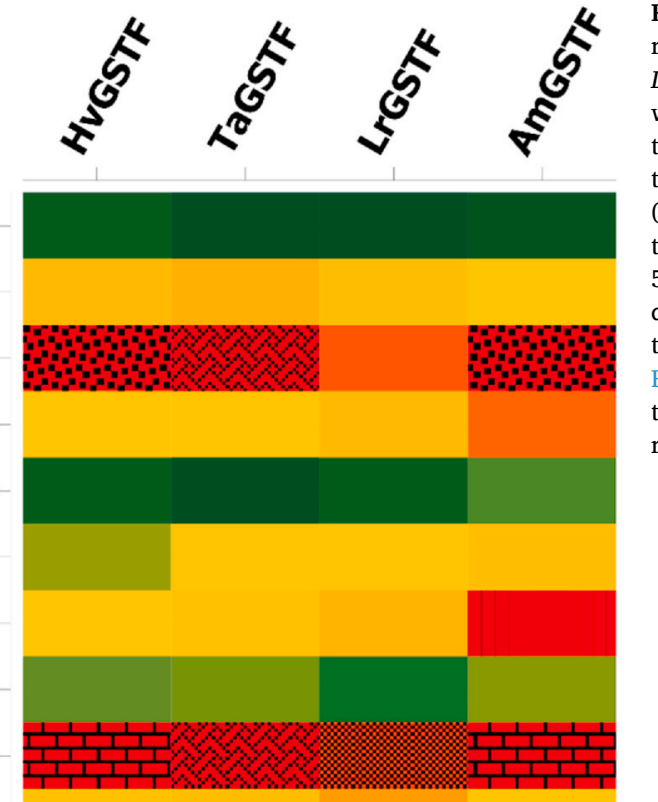

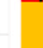
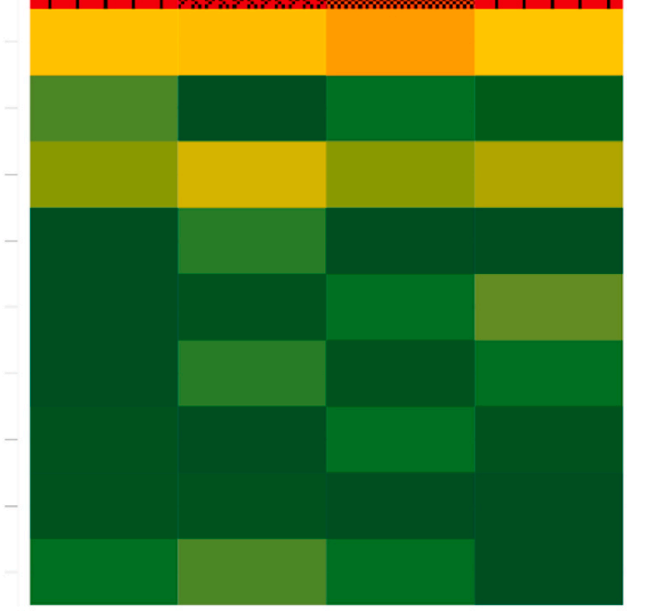

Inhibition (\%)
Fig. 2. Inhibition potency of selected natural products towards $H \nu \mathrm{GSTF}, \mathrm{TaGSTF}$, LrGSTF and AmGSTF. The enzyme assays were implemented in triplicate employing the GSH/CDNB system. The colors represent the mean values of three inhibition assays (\%) for each natural product against the tested enzymes with variation of less than $5 \%$ in all cases. The motifs allow the discrimination for specific inhibition potency according to the table legend shown in Fig. 1. (For interpretation of the references to color in this figure legend, the reader is referred to the Web version of this article.)

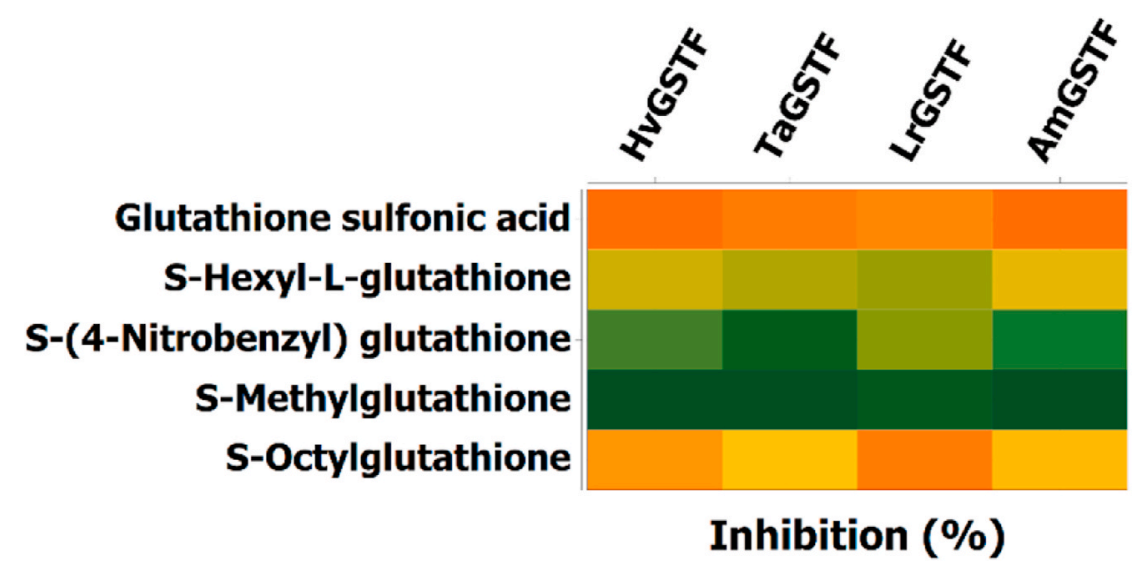

Fig. 3. Inhibition potency of selected S-alkyl-GSH inhibitors towards $H v$ GSTF, TaGSTF, GSTF, LrGSTF and AmGSTF. The enzyme assays were implemented in triplicate employing the GSH/CDNB system. The colors represent the mean values of three inhibition assays (\%) for each S-alkyl-GSH inhibitor against the tested enzymes with variation less than $5 \%$ in all cases. The motifs allow the discrimination for specific inhibition potency according to the table legend shown in Fig. 1. (For interpretation of the references to color in this figure legend, the reader is referred to the Web version of this article.) houses the highly variable and hydrophobic substrate-binding $\mathrm{H}$-site (Fig. 5A and B).

All molecules have S-(p-nitrobenzyl)-glutathione (Nb-GSH) bound at the active site, apart from molecule D which has a bound GSH moiety instead. A glycerol molecule is present at the G-site of all molecules. A second glycerol is near the nitro group of Nb-GSH in molecule A but not in all molecules. The interaction study between the enzyme molecules reveals that the interface area of A-E and C-F dimers is $\sim 1407 \AA^{2}$. Other interfaces are significantly smaller (A-C $\sim 612.5 \AA^{2}$ interface area; A-D $569.3 \AA^{2}, \mathrm{~B}-\mathrm{F} \sim 469.8 \AA^{2}$ ). Furthermore, between molecules A-E, C-F, B- 
A

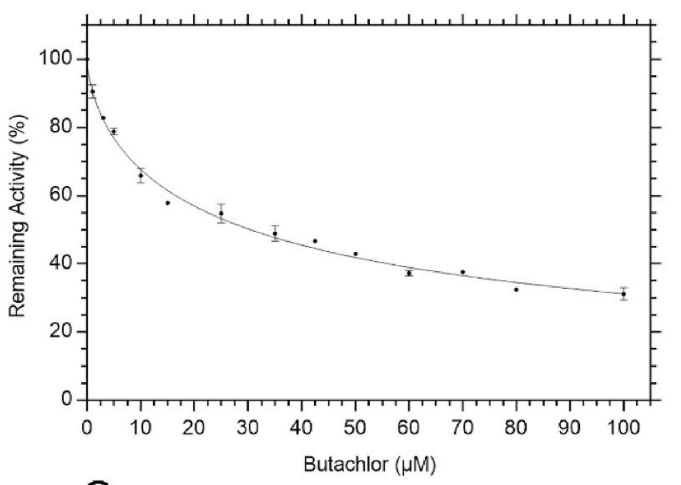

C

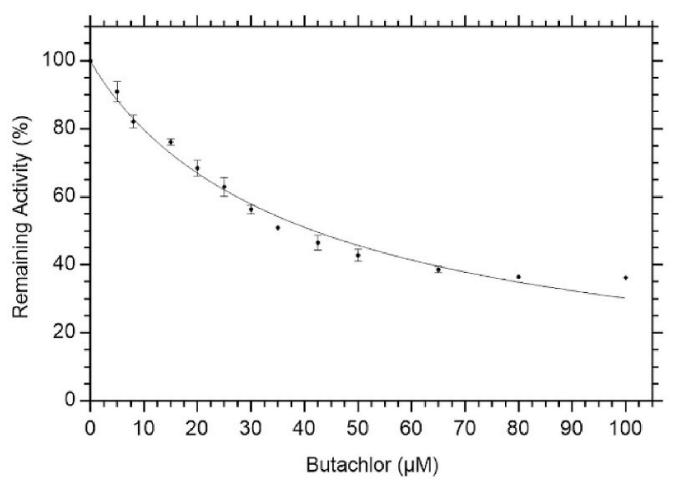

B

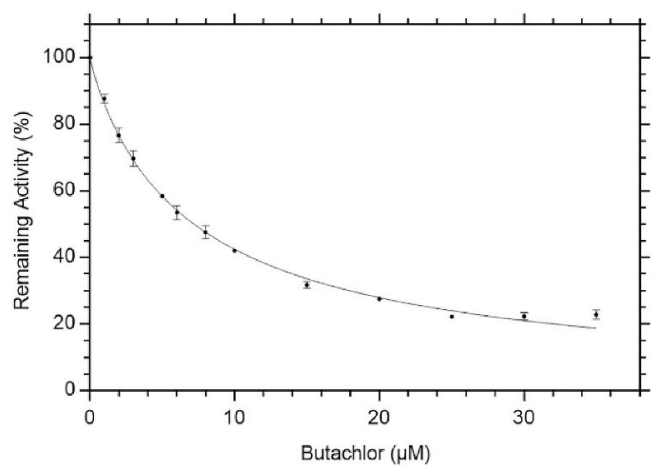

D

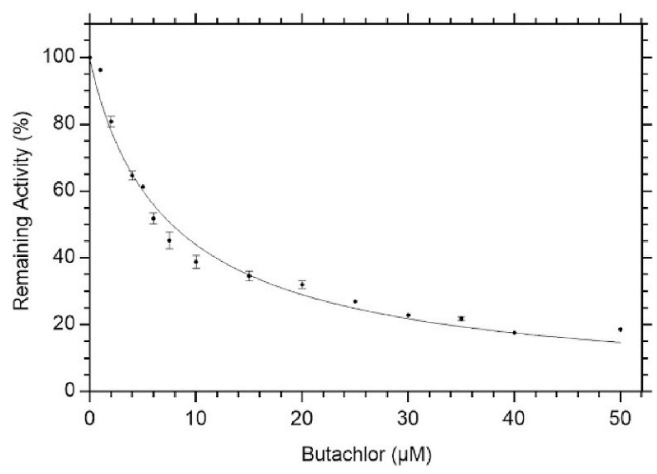

Fig. 4. Concentration-response curves for the determination of $\mathrm{IC}_{50}$ values for butachlor with (A) $H v G S T F$, (B) $L r G S T F$, (C) TaGSTF and (D) AmGSTF.

D, A-C, A-D, C-E and B-F 9, 8, 9, 7, 2, 2 and 1 salt bridge are spotted while the number of hydrogen bonds between the subunits are $2,1,2,7$, $3,1,3$ respectively.

\subsection{Comparison of the LrGSTF crystal structure with its homologue AmGSTF}

The LrGSTF monomer is superimposed and aligned with AmGSTF with a root mean square deviation (RMSD) of $0.722 \AA$ (sequence identity $89.4 \%$ ). The RMSD value suggests a close structural similarity between the two enzymes (Fig. 5A and B). Eleven substitutions are found in the variable C-terminal domain and 9 at the N-terminal domain (Fig. 5C). The analysis showed that the catalytic -STNV motif at the G-site of the enzyme is well conserved as well as the residues involved in $\mathrm{H}$-site of the enzyme are identical to AmGSTF. A non-polar and bulky Phe21 in LrGSTF is found to replace Cys21 in AmGSTF. A noticeable difference between the structures is observed towards the end of $\alpha 4$ helix which bends slightly towards Nb-GSH at the H-site of the enzyme (Fig. 5A).

In both $L r G S T F$ and AmGSTF enzyme complexes, GSH is bound at the same position and in similar conformation in the cleft between the Cand N-terminal domains (Fig. 5A and B). The nitrobenzyl moiety in NbGSH occupies the H-site hydrophobic pocket of LrGSTF where hydrophobic electrophiles usually bind, thus acting as an enzyme inhibitor. The sulfur group of Nb-GSH is found to be located $2.78 \AA$ from the amide group of Asn14, allowing a hydrogen bond to be formed. The structural superposition between the two GSTFs allows visualization of the conformational changes the GSTFs undergo upon substrate binding. The residue Met126 which is oriented away from the active site in LrGSTF owing to the binding of the bulky nitrobenzyl group is found pointing towards the H-site in AmGSTF and actively involved in van de Waals interaction with succinic acid (Fig. 6. B, C). Moreover, the nitro-benzyl ring of Nb-GSH is involved in two $\pi-\pi$ interactions with Phe122 and Tyr118 residues of the enzyme. Although the aromatic ring of Phe122 is similar in the two enzyme complexes, possibly owing to the bound succinate in AmGSTF, its position in molecule D of LrGSTF which has only GSH bound points towards the $\mathrm{H}$-site. Upon Nb-GSH binding, Phe122 swings away to optimize interactions and provide space for the nitro-benzyl group of Nb-GSH. Tyr118 shows a different position in subunit $\mathrm{C}$ although it retains a similar position in all other subunits, suggesting some flexibility. Met126 is found to interact via weak van der Waals with the nitro-benzyl ring. The guanidinium group of Arg127 interacts with the nitroxide group of the nitrobenzyl ring. Residues Lys42, Gln54, Ile55, Glu67 and Ser68 are involved in the formation of Hbond interactions with Nb-GSH. Other residues such as Ser12, Thr13, Asn14, Phe36, His41, Gly53, Arg69 interact via weak non-bonding van der Waals forces with the Nb-GSH moiety. These interacting residues with Nb-GSH are found to be strictly conserved in AmGSTF as well (Fig. 5C).

The molecules C and D of LrGSTF along with the bound Nb-GSH and GSH were superimposed with RMSD value of $0.41 \AA$ (Fig. 6D). The structural superposition shows that the binding of enzyme inhibitor like $\mathrm{Nb}$-GSH reduces the active site opening, thus limiting the accessibility of the substrates to the active site. A slight movement $(\sim 2.0 \AA)$ is observed at the beginning of helix $\alpha 2$. It is found that movement of $\alpha 2$ and $\alpha 4$ towards each other (Fig. 6D) helps to narrow the accessibility to the active site. Instead, molecule D with bound GSH possess comparatively wider gateway to the active site cavity for substrate binding to the $\mathrm{H}$-site of the active site. Overall, based upon the RMSD values and structural alignment it can be concluded that ligand binding is not accompanied by significant changes in the conformation of these GSTF enzymes. However, changes in individual residues appear necessary for efficient binding.

\subsection{Structure-based $3 D$ pharmacophore design method}

The homology modelling of the HvGSTF and TaGSTF was carried out using the Modeller package (version 9.10) (Sali et al., 1995). The sequence identity between the query GST sequences and the chosen 
A

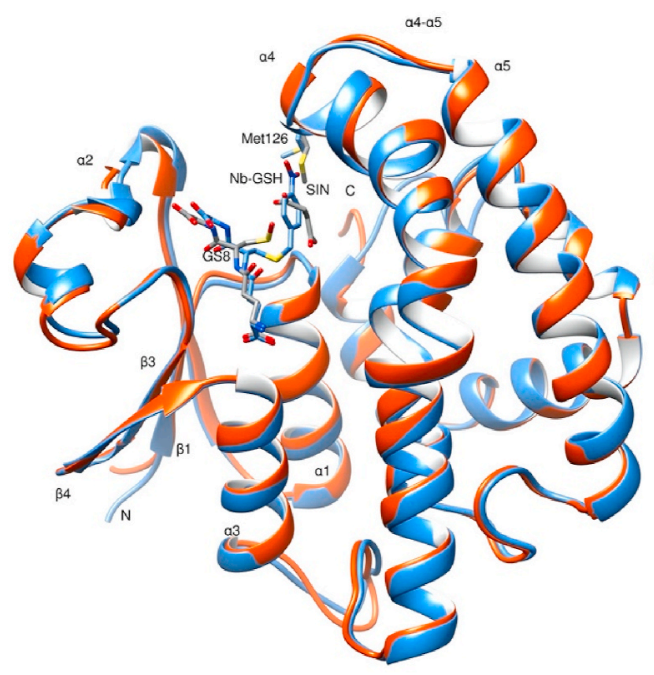

B

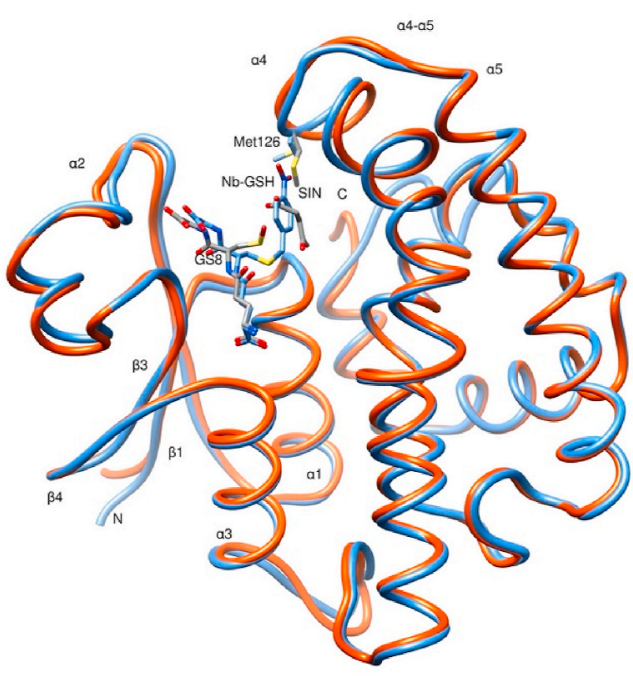

Fig. 5. A. Superposition of LrGSTF monomer (colored navy blue) on AmGSTF (colored orange red). Nb-GSH (backbone colored blue) and S-hydroxy-GSH (GS8, colored gray) molecules bound to the G-sites of LrGSTF and AmGSTF is displayed. Succinic acid (SIN) bound to the $\mathrm{H}$-site of AmGSTF is colored gray. B. A cartoon representation of the superimposition for clarity. C. Structure-based sequence alignment of $L r G S T F$ and AmGSTF. The $L r G S T F$ numbering is shown above the alignment and conserved areas are shown shaded. Residues involved in Nb-GSH binding are marked with triangles and those that make $\mathrm{H}$-bonds at the dimer interface in blue stars. The red triangles identify residues interacting with the N-nitro benzyl group of Nb-GSH in LrGSTF, these residues also correspond with residues in AmGSTF that interact with succinic acid in the $\mathrm{H}$-site. (For interpretation of the references to color in this figure legend, the reader is referred to the Web version of this article.)

C
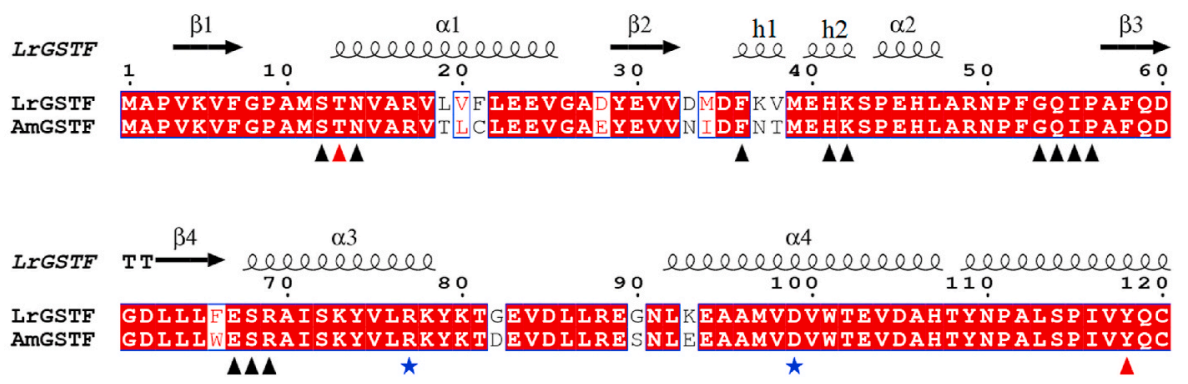

$\alpha 5$

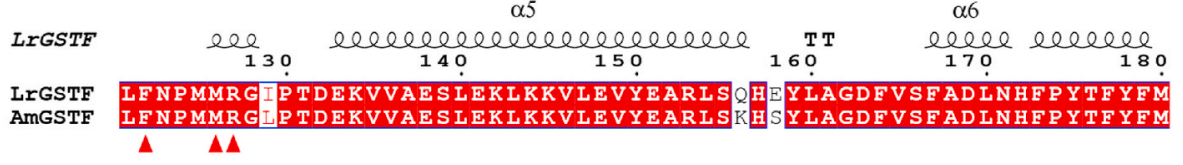
$\alpha 6$

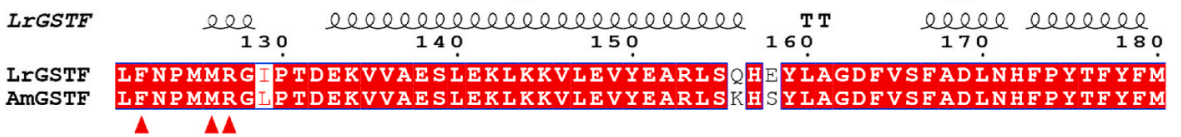

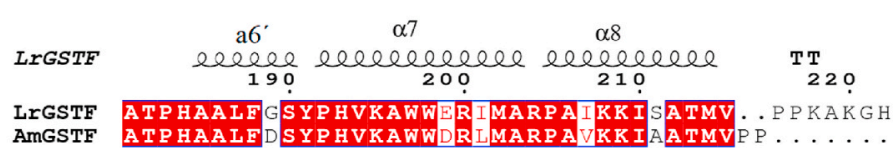

crystal structure template was at the lowest at least $80 \%$. That allowed for conventional homology modelling to be considered. The x-ray template structure of $L r G S T F$ is a GST co-crystallized with Nb-GSH. Therefore, the catalytic, physical and physicochemical properties of the active site of each modeled GST was initially assessed based on their potential to accommodate Nb-GSH. It was found that the two modeled GSTs were not able to fully fit the Nb-GSH molecule in their catalytic site (Fig. 7). The catalytic sites of $H v$ GSTF and TaGSTF were slightly smaller and tighter compared to the two crystal structures (LrGSTF and AmGSTF). Both crystal structures and both models were energetically minimized and subjected to molecular dynamics simulations in the presence of $\mathrm{Nb}-\mathrm{GSH}$. The interaction maps of HvGSTF, TaGSTF and AmGSTF to the Nb-GSH substrate was used to identify the variable and the dynamic part of each modeled catalytic site in comparison to the template structure ( $L r G S T F)$, for the optimal annotation of the conserved part of each catalytic site and the consecutive designing of the structure-based pharmacophore model.

For the training of the pharmacophore model, a variety of physicochemical descriptors were used. The volume, size and rigidity of the compounds as a determining factor of biological activity is demonstrated in Fig. 8. It can be easily concluded that the more potent butachlor molecule is much closer to the physical and physicochemical arrangement of $\mathrm{Nb}-\mathrm{GSH}$, when compared to the poorer inhibitor, for example endosulfan. Using a hybrid pharmacophore design approach, a collective pharmacophore model was established for the four GSTFs (LrGSTF, AmGSTF, TaGSTF and HvGSTF). 3D Pharmacophore designing methods take into account both the three-dimensional structures and binding modes of receptors and inhibitors, in order to identity regions that are favorable or not for a specific receptor-inhibitor interaction. The description of the receptor-inhibitor interaction pattern is determined by a correlation between the characteristic properties of the inhibitors and their biochemically determined enzymatic activity.

A 3D pharmacophore was generated for the active site of each GSTF, as a molecular model that ensembles all steric and electronic features that are necessary to ensure optimal covalent and non-covalent interactions with GST. The pharmacophoric features investigated, 
A

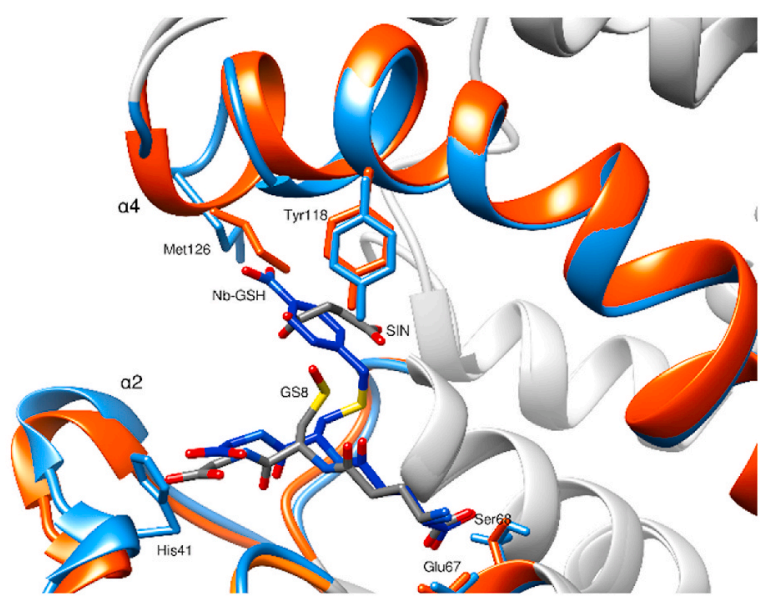

B
Fig. 6. A. Comparison of $\alpha 4$ and $\alpha 2$ helices in $L r$ GSTF and AmGSTF. LrGSTF is colored blue and AmGSTF in orange. S-hydroxy-GSH (GS8) bound to the G-site of AmGSTF is colored dark gray for good visual. The $\mathrm{H}-$ site of $L r G S T F$ is occupied by nitro-benzyl ring of NbGSH bordered by shortened $\alpha 4$ helix as compared to AmGSTF. The conformational change in $\alpha 4$ helix in AmGSTF is shown to accommodate succinic acid (SIN). B. Active site residues comparisons of AmGSTF C. Active site residues comparisons of LrGSTF. The bound ligands GS8, SIN and Nb-GSH are colored in dark gray. D. Comparison of molecule C (red) and molecule D (blue) of LrGSTF. The enzyme backbone is shown in molecule trace diagram. Nb-GSH and GSH bound in the active site cavities of molecule $\mathrm{C}$ and molecule D, respectively, are shown in stick representation and labelled. (For interpretation of the references to color in this figure legend, the reader is referred to the Web version of this article.)
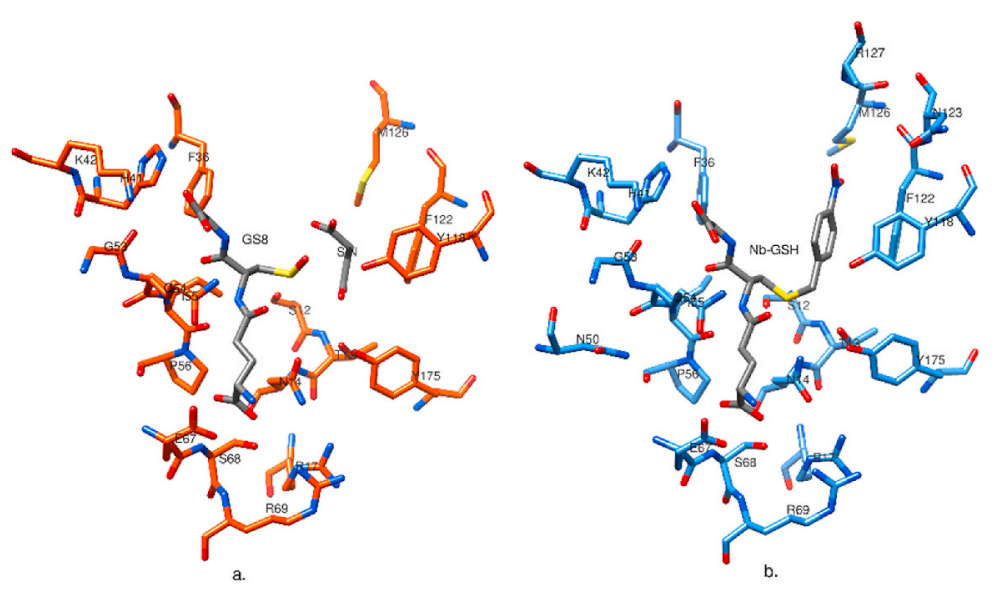

C

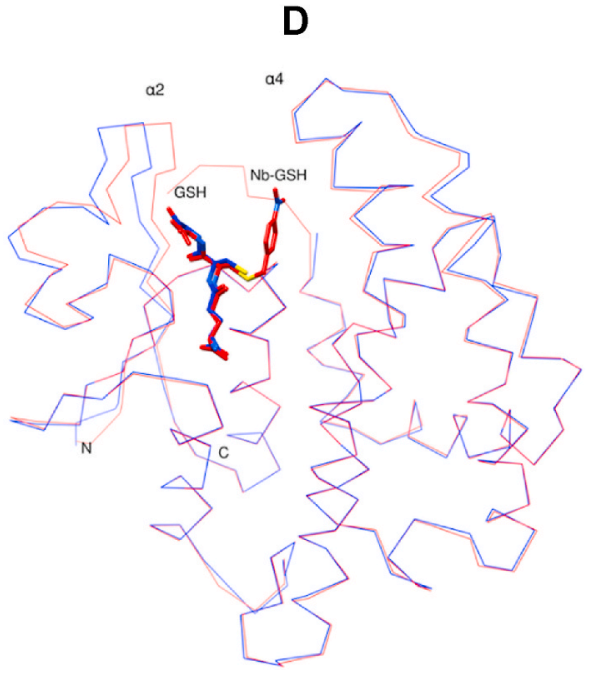

included positively or negatively ionized regions, hydrogen bond donors and acceptors, aromatic regions and hydrophobic areas. Pharmacophore hypotheses showing best correlation in the 3D arrangement of features in a given training set of compounds with the corresponding pharmacological activities are formed and ranked. In particular, the amino acids of all four GSTFs in this study that interact with Nb-GSH, plus the nearby supporting amino acids were evaluated for their potential to be exploited as unique per GST, thus constituting possible pharmacological targets. In this direction, the four GSTF primary sequences were aligned and the abovementioned amino acids were highlighted (Suppl. Figure 1). The core consisted of the residues that are in direct or indirect interaction to Nb-GSH in both crystal structures (Suppl. Table 1). It was found that there is a set of Tyr residues that are necessary for the optimal interaction to all GSTs. The most prevalent in interaction to Nb-GSH in 


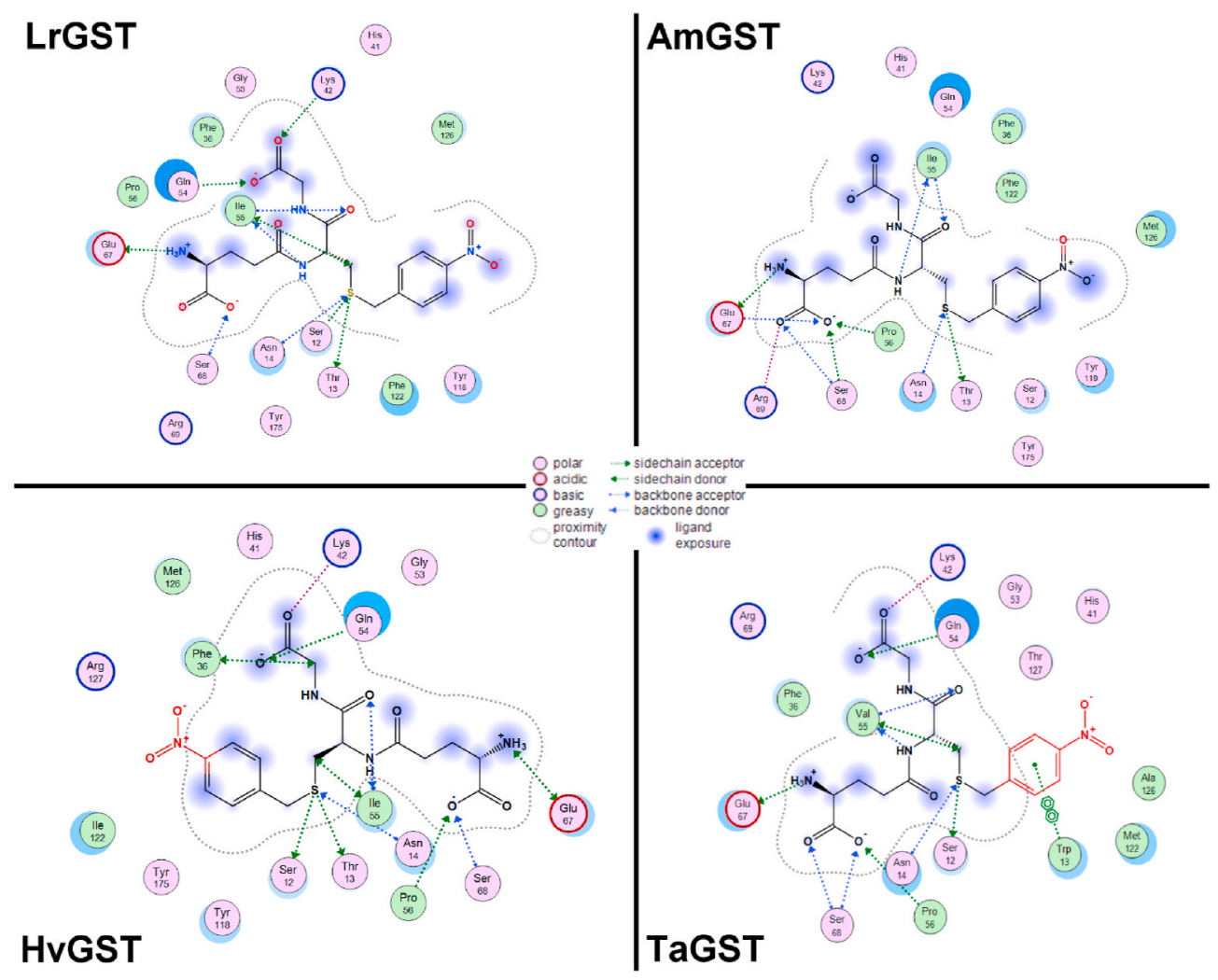

Fig. 7. The molecular interactions between the Nb-GSH molecule and the four studied GSTs (LrGSTF, AmGSTF, HvGSTF and TaGSTF).
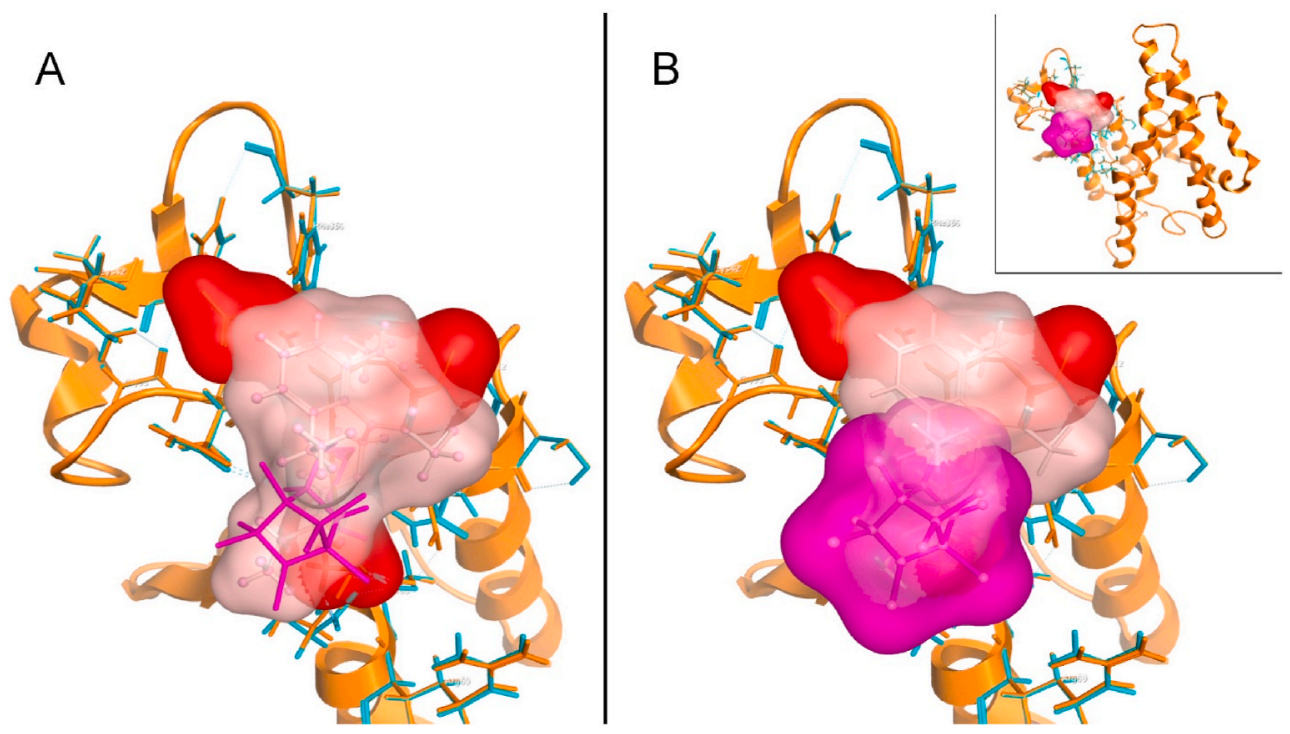

Fig. 8. Close-up view of Nb-GSH, butachlor and endosulfan binding to LrGSTF. The Nb-GSH is shown in red cloud while the docked butachlor (best inhibitor) is shown in gray cloud representation. The docking conformation of endosulfan (a weak inhibitor) is likewise represented in magenta cloud. (For interpretation of the references to color in this figure legend, the reader is referred to the Web version of this article.)

this pharmacophore model was found to be Tyr118. This residue is well conserved but its optimal positioning in the three-dimensional conformation of the catalytic site of the GSTs was found to be dependent on an outer patch of adjacent residues. Those residues span from position 132 to 134 and for both LrGSTF and AmGSTF the sequence is DEK. On the other hand, $H \nu$ GSTF and TaGSTF have instead of DEK, NQT and IDD, respectively. The loss of this motif seems to destabilize the optimal positioning of Tyr118 and thus significantly altering the inhibition potential of HvGSTF and TaGSTF to the given compounds.
Taking into account the interaction potential and contribution of each involved residue position, the GSTF specific pharmacophore was designed (Fig. 9A). This pharmacophore model consists of four annotation points that can be used to describe the properties of the most potent and promising compounds in this study. Namely, there are two Hacceptor groups (green bubbles), an aromatic one (brown bubble) and an aliphatic one (pink bubble) as shown in Fig. 9A. The pharmacophore model was assessed by its potential to accommodate Nb-GSH (Fig. 9B). Overall, we propose that this pharmacophore model can be used for the 
A
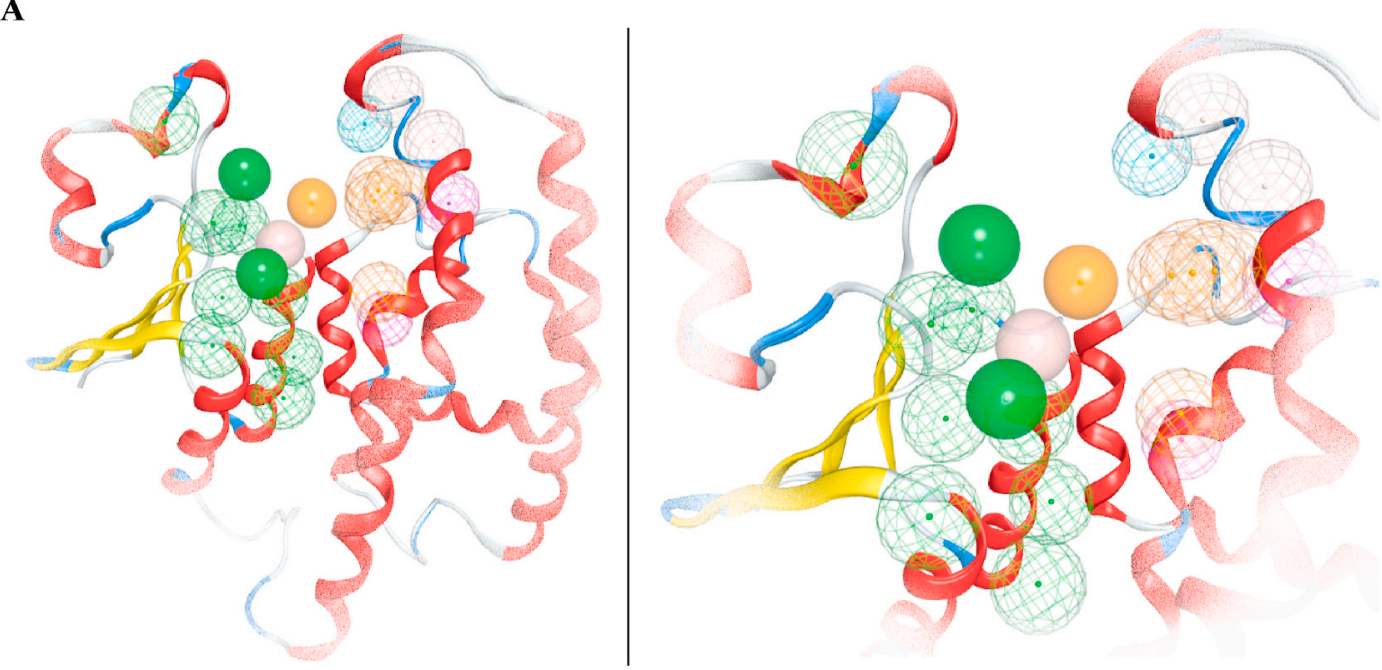

B

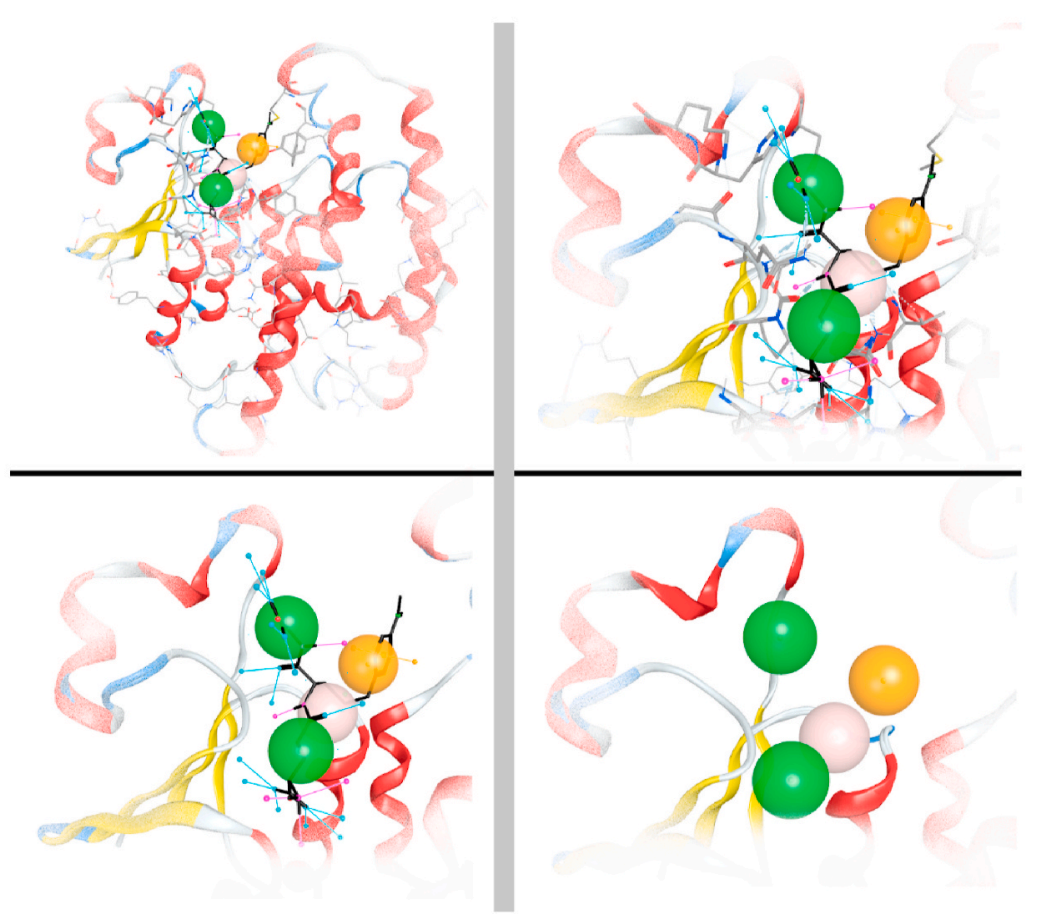

Fig. 9. A. The designed pharmacophore model (solid spheres) and the pharmacophoric annotations of the GSTFs that were used to design it (wire spheres). The pharmacophore model consists of four points that can be used to describe the properties of the most potent and promising compounds in this study. Green bubbles represent $\mathrm{H}$-acceptor groups, brown bubble represents an aromatic group and the pink bubble represents an aliphatic group. B. The pharmacophore model of Fig. 9A in the presence of the Nb-GSH molecule. Color representation follows the conventions of Fig. 9A. (For interpretation of the references to color in this figure legend, the reader is referred to the Web version of this article.)

fast and efficient screening of potential modulators of this family of GST enzymes.

\section{Conclusions}

MHR-GSTFs are important targets for chemical intervention in resistant weed management, using specific inhibitors selected from the available pesticide databases. The herbicide butachlor is a selective and potent inhibitor towards MHR-GSTF from weeds and could be used in combination with other herbicides to reverse the MHR phenotype. Crystal structure analysis of $L r G S T F$ revealed that the conserved residue Tyr118 plays important role in ligand binding and pharmacophore design. A set of residues appears to modulate the optimal positioning of Tyr118 and thus altering the sensitivity of $\mathrm{H} v \mathrm{GSTF}$ and TaGSTF towards butachlor. Butachlor is a registered herbicide and can be used in the field, without the necessity of additional regulations. Taking into account the rapid spread of MHR in grass weeds and the restricted development of new herbicides, the approach that we used in this study offers a viable alternative strategy in counteracting herbicide resistance in the field.

\section{Contribution}

NG, NP and DV performed the experiments under the supervision of ACP and NEL. All authors contributed to the writing of the manuscript, have read and approved the final manuscript.

\section{Declaration of competing interest}

The authors declare that they have no known competing financial 
interests or personal relationships that could have appeared to influence the work reported in this paper.

\section{Acknowledgements}

NG acknowledges support by the Hellenic Foundation for Research and Innovation (HFRI) and the General Secretariat for Research and Technology (GSRT), under the HFRI PhD Fellowship grant (GA. No. 8904/22-09-2017). NP and ACP thank Charlène Le Meur for help with the crystallization trials and Johanna Hakanpää at EMBL-Hamburg for help during data collection. Access to EMBL-Hamburg (c/o DESY) P14 beamline was provided by iNEXT, project number 653706, funded by the European Union. Infrastructure support from Biocenter Finland is acknowledged.

\section{Appendix A. Supplementary data}

Supplementary data to this article can be found online at https://doi. org/10.1016/j.plaphy.2020.11.018.

\section{References}

Adams, P.D., Afonine, P.V., Bunkóczi, G., Chen, V.B., Davis, I.W., Echols, N., Headd, J.J., Hung, L.-W., Kapral, G.J., Grosse-Kunstleve, R.W., McCoy, A.J., Moriarty, N.W., Oeffner, R., Read, R.J., Richardson, D.C., Richardson, J.S., Terwilliger, T.C., Zwart, P.H., 2010. PHENIX: a comprehensive Python-based system for macromolecular structure solution. Acta Crystallogr. D Biol. Crystallogr. 66, 213-221.

Ahmad, L., Rylott, E.L., Bruce, N.C., Edwards, R., Grogan, G., 2017. Structural evidence for Arabidopsis glutathione transferase AtGSTF2 functioning as a transporter of small organic ligands. FEBS Open Bio 7, 122-132.

Axarli, I.A., Rigden, D.J., Labrou, N.E., 2004. Characterization of the ligandin site of maize glutathione S-transferase I. Biochem. J. 382, 885-893.

Axarli, I., Dhavala, P., Papageorgiou, A.C., Labrou, N.E., 2009a. Crystal structure of Glycine max glutathione transferase in complex with glutathione: investigation of the mechanism operating by the Tau class glutathione transferases. Biochem. J. 422, 247-256.

Axarli, I., Dhavala, P., Papageorgiou, A.C., Labrou, N.E., 2009b. Crystallographic and functional characterization of the fluorodifen-inducible glutathione transferase from Glycine max reveals an active site topography suited for diphenylether herbicides and a novel L-site. J. Mol. Biol. 385, 984-1002.

Benekos, K., Kissoudis, C., Nianiou-Obeidat, I., Labrou, N., Madesis, P., Kalamaki, M., Makris, A., Tsaftaris, A., 2010. Overexpression of a specific soybean GmGSTU4 isoenzyme improves diphenyl ether and chloroacetanilide herbicide tolerance of transgenic tobacco plants. J. Biotechnol. 150, 195-201.

Bradford, M.M., 1976. A rapid and sensitive method for the quantitation of microgram quantities of protein utilizing the principle of protein-dye binding. Anal. Biochem. 1 $-2,248-254$.

Bunkoczi, G., Read, R.J., 2011. Improvement of molecular-replacement models with Sculptor. Acta Crystallogr. D Biol. Crystallogr. 67, 303-312.

Busi, R., Porri, A., Gaines, T.A., Powles, S.B., 2018. Pyroxasulfone resistance in Lolium rigidum is metabolism-based. Pestic. Biochem. Physiol. 148, 74-80.

Chen, R., Li, L., Weng, Z., 2003. ZDOCK: an initial-stage protein-docking algorithm. Proteins 52, 80-87.

Cho, H.-Y., Lee, H.Y., Kong, K.-H., 2007. A phi class glutathione S-transferase from Oryza sativa (OsGSTF5): molecular cloning, expression and biochemical characteristics. J. Biochem. Mol. Biol. 40, 511-516.

Chronopoulou, E.G., Papageorgiou, A.C., Markoglou, A., Labrou, N.E., 2012. Inhibition of human glutathione transferases by pesticides: development of a simple analytical assay for the quantification of pesticides in water. J. Mol. Catal. B Enzym. 81, 43-51.

Cummins, I., Cole, D.J., Edwards, R., 1999. A role for glutathione transferases functioning as glutathione peroxidases in resistance to multiple herbicides in blackgrass. Plant J. 18, 285-292.

Cummins, I., O'Hagan, D., Jablonkai, I., Cole, D.J., Hehn, A., Werck-Reichhart, D., Edwards, R., 2003. Cloning, characterization and regulation of a family of phi class glutathione transferases from wheat. Plant Mol. Biol. 52, 591-603.

Cummins, I., Wortley, D.J., Sabbadin, F., He, Z., Coxon, C.R., Straker, H.E., Sellars, J.D. Knight, K., Edwards, L., Hughes, D., Kaundun, S.S., Hutchings, S.J., Steel, P.G., Edwards, R., 2013. Key role for a glutathione transferase in multiple-herbicide resistance in grass weeds. Proc. Natl. Acad. Sci. U.S.A. 110, 5812-5817.

Dixon, D.P., Edwards, R., 2009. Selective binding of glutathione conjugates of fatty acid derivatives by plant glutathione transferases. J. Biol. Chem. 284, 21249-21256.

Dixon, D.P., Edwards, R., 2018. Protein-ligand fishing in planta for biologically active natural products using glutathione transferases. Front. Plant Sci. 9, 1659.
Dixon, D.P., Sellars, J.D., Edwards, R., 2011. The Arabidopsis phi class glutathione transferase AtGSTF2: binding and regulation by biologically active heterocyclic ligands. Biochem. J. 438, 63-70.

Dixon, D.P., Skipsey, M., Edwards, R., 2010. Roles for glutathione transferases in plant secondary metabolism. Phytochemistry 71, 338-350.

Dobritzsch, D., Grancharov, K., Hermsen, C., Krauss, G.-J., Schaumlöffel, D., 2020. Inhibitory effect of metals on animal and plant glutathione transferases. J. Trace Elem. Med. Biol. 57, 48-56.

Eisenberg, D., Lüthy, R., Bowie, J.U., 1997. VERIFY3D: assessment of protein models with three-dimensional profiles. Methods Enzymol. 277, 396-404.

Evans, P.R., Murshudov, G.N., 2013. How good are my data and what is the resolution? Acta Crystallogr. D Biol. Crystallogr. 69, 1204-1214.

Gaines, T.A., Duke, S.O., Morran, S., Rigon, C.A.G., Tranel, P.J., Küpper, A., Dayan, F.E., 2020. Mechanisms of evolved herbicide resistance. J. Biol. Chem. 295, 10307-10330.

Gao, S., Liu, Y.-Y., Jiang, J.-Y., Fu, Y., Zhao, L.-X., Li, C.-Y., Ye, F., 2019. Protective Responses induced by chiral 3-dichloroacetyl oxazolidine safeners in maize (Zea mays L.) and the detoxification mechanism. Molecules 24, 3060.

Georgakis, N., Poudel, N., Papageorgiou, A.C., Labrou, N.E., 2020. Comparative structural and functional analysis of Phi class glutathione transferases involved in Multiple-Herbicide Resistance of grass weeds and crops. Plant Physiol. Biochem. 149, 266-276.

Hess, B., Kutzner, C., van der Spoel, D., Lindahl, E., 2008. GROMACS 4: algorithms for highly efficient, load-balanced, and scalable molecular simulation. J. Chem. Theor. Comput. 4, 435-447.

Incardona, M.-F., Bourenkov, G.P., Levik, K., Pieritz, R.A., Popov, A.N., Svensson, O. 2009. EDNA: a framework for plugin-based applications applied to X-ray experiment online data analysis. J. Synchrotron Radiat. 16, 872-879.

Kabsch, W., 2010. XDS. Acta Crystallogr. D Biol. Crystallogr. 66, 125-132.

Karatolos, N., Williamson, M.S., Denholm, I., Gorman, K., ffrench-Constant, R.H., Bass, C., 2012. Over-expression of a cytochrome P450 is associated with resistance to pyriproxyfen in the greenhouse whitefly Trialeurodes vaporariorum. PloS One 7 (2), e31077.

Kitamura, S., Matsuda, F., Tohge, T., Yonekura-Sakakibara, K., Yamazaki, M., Saito, K., Narumi, I., 2010. Metabolic profiling and cytological analysis of proanthocyanidins in immature seeds of Arabidopsis thaliana flavonoid accumulation mutants. Plant J. $62,549-559$.

Laskowski, R.A., Rullmannn, J.A., MacArthur, M.W., Kaptein, R., Thornton, J.M., 1996. AQUA and PROCHECK-NMR: programs for checking the quality of protein structures solved by NMR. J. Biomol. NMR 8, 477-486.

Matthews, B.W., 1968. Solvent content of protein crystals. J. Mol. Biol. 33, 491-497.

McCoy, A.J., Grosse-Kunstleve, R.W., Adams, P.D., Winn, M.D., Storoni, L.C., Read, R.J., 2007. Phaser crystallographic software. J. Appl. Crystallogr. 40, 658-674.

Molecular Operating Environment (MOE), 2020. 2019.01; chemical computing group ULC, 1010 sherbooke st. West, suite \#910, montreal, QC, Canada, H3A 2R7, 2020. http://www.chemcomp.com.

Nakka, S., Godar, A.S., Thompson, C.R., Peterson, D.E., Jugulam, M., 2017. Rapid detoxification via glutathione S-transferase (GST) conjugation confers a high level of atrazine resistance in Palmer amaranth (Amaranthus palmeri). Pest Manag. Sci. 73, 2236-2243.

Neuefeind, T., Huber, R., Dasenbrock, H., Prade, L., Bieseler, B., 1997. Crystal structure of herbicide-detoxifying maize glutathione S-transferase-I in complex with lactoylglutathione: evidence for an induced-fit mechanism. J. Mol. Biol. 274, 446-453.

Pégeot, H., Koh, C.S., Petre, B., Mathiot, S., Duplessis, S., Hecker, A., Didierjean, C., Rouhier, N., 2014. The poplar Phi class glutathione transferase: expression, activity and structure of GSTF1. Front. Plant Sci. 5, 712.

Pégeot, H., Mathiot, S., Perrot, T., Gense, F., Hecker, A., Didierjean, C., Rouhier, N. 2017. Structural plasticity among glutathione transferase Phi members: natural combination of catalytic residues confers dual biochemical activities. FEBS J. 284 2442-2463.

Sali, A., Potterton, L., Yuan, F., van Vlijmen, H., Karplus, M., 1995. Evaluation of comparative protein modeling by MODELLER. Proteins 23, 318-326.

Skopelitou, K., Muleta, A.W., Papageorgiou, A.C., Chronopoulou, E.G., Pavli, O., Flemetakis, E., Skaracis, G.N., Labrou, N.E., 2017. Characterization and functional analysis of a recombinant tau class glutathione transferase GmGSTU2-2 from Glycine max. Int. J. Biol. Macromol. 94, 802-812.

Sylvestre-Gonon, E., Law Simon, R., Schwartz, M., Robe, K., Keech, O., Didierjean, C., Dubos, C., Rouhier, N., Hecker, A., 2019. Functional, structural and biochemical features of plant serinyl-glutathione transferases. Front. Plant Sci. 10, 608.

Sylvestre-Gonon, E., Schwartz, M., Girardet, J.-M., Hecker, A., Rouhier, N., 2020. Is there a role for tau glutathione transferases in tetrapyrrole metabolism and retrograde signalling in plants? Phil. Trans. R. Soc. B 375, 20190404.

Tétard-Jones, C., Sabbadin, F., Moss, S., Hull, R., Neve, P., Edwards, R., 2018. Changes in the proteome of the problem weed blackgrass correlating with multiple-herbicide resistance. Plant J. 94, 709-720.

Zhao, J., 2015. Flavonoid transport mechanisms: how to go, and with whom. Trends Plant Sci. 20, 576-585. 